\title{
THE SPECTRAL MAPPING PROPERTY FOR $p$-MULTIPLIER OPERATORS ON COMPACT ABELIAN GROUPS
}

\author{
WERNER J. RICKER
}

(Received 1 July 2003; revised 9 January 2004)

Communicated by $\mathrm{G}$. Willis

\begin{abstract}
Let $G$ be a compact abelian group and $1<p<\infty$. It is known that the spectrum $\sigma\left(T_{\psi}\right)$, of a Fourier $p$-multiplier operator $T_{\psi}$ acting in $L^{p}(G)$, may fail to coincide with its natural spectrum $\overline{\psi(\Gamma)}$ if $p \neq 2$; here $\Gamma$ is the dual group to $G$ and the bar denotes closure in $C$. Criteria are presented, based on geometric, topological and/or algebraic properties of the compact set $\sigma\left(T_{\psi}\right)$, which are sufficient to ensure that the equality $\sigma\left(T_{\psi}\right)=\overline{\psi(\Gamma)}$ holds.
\end{abstract}

2000 Mathematics subject classification: primary 43A22, 47A10.

\section{Introduction}

Let $G$ be a compact abelian group with (discrete) dual group $\Gamma$. The Fourier transform

$$
\hat{f}(\gamma):=\int_{G} f(g)\langle-g, \gamma\rangle d g, \quad \gamma \in \Gamma
$$

is defined for all $f \in L^{1}(G)$. According to Hausdorff-Young's inequality, the Fourier transform map $f \mapsto \hat{f}$ is linear and continuous from $L^{p}(G)$ into $\ell^{p^{\prime}}(\Gamma)$, where $1 / p+1 / p^{\prime}=1$ and $1 \leq p \leq 2$. In the above formula for $\hat{f}$, replacing $f(g) d g$ with $d \mu(g)$ gives the definition of the Fourier-Stieltjes transform $\hat{\mu}: \Gamma \rightarrow \mathbb{C}$ for any finite regular Borel measure $\mu$ on $G$. An element $T$ from $\mathscr{L}\left(L^{p}(G)\right)$, the Banach algebra of all continuous linear operators from $L^{p}(G)$ into itself, is called a (Fourier) p-multiplier operator if it commutes with each translation operator $\tau_{h}$, for $h \in G$, where $\tau_{h} f: g \mapsto f(g-h)$. Equivalently, there exists $\psi \in \ell^{\infty}(\Gamma)$, necessarily

(C) 2005 Australian Mathematical Society $1446-7887 / 05 \$ A 2.00+0.00$ 
unique, such that $\widehat{T f}=\psi \hat{f}$ for $f \in L^{2} \cap L^{p}(G)$. The function $\psi: \Gamma \rightarrow \mathbb{C}$ is called a $p$-multiplier for $G$ and the corresponding operator $T$ is denoted by $T_{\psi}$; the $p$-dependence of $T_{\psi}$ is suppressed since $p$ will always be clearly identified. The space of all $p$-multipliers for $G$ is denoted by $\mathscr{M}^{p}(G) \subseteq \ell^{\infty}(\Gamma)$ and the space of all $p$-multiplier operators by $O_{p}(G) \subseteq \mathscr{L}\left(L^{p}(G)\right)$. The inequality

$$
\|\psi\|_{\ell^{x}(\Gamma)} \leq\left\|T_{\psi}\right\|_{\mathscr{L}\left(L^{p}(G)\right)}, \quad \psi \in \mathscr{M}^{p}(G)
$$

is well known. If we equip $\mathscr{M}^{p}(G)$ with the norm $\|\psi\|_{p}:=\left\|T_{\psi}\right\|_{\mathscr{L}_{\left(L^{p}(G)\right)} \text {, then }}$ $\mathscr{M}^{p}(G)$ is a unital commutative semisimple Banach algebra for pointwise multiplication. For each $\psi \in \mathscr{M}^{p}(G)$, the functions $\operatorname{Re}(\psi), \operatorname{Im}(\psi)$ and $\bar{\psi}$ (the complex conjugate of $\psi$ ) also belong to $\mathscr{M}^{p}(G)$ with $\psi \mapsto \bar{\psi}$ being an isometric involution on $\mathscr{M}^{p}(G)$. Since $\mathscr{M}^{p}(G)$ is isometrically isomorphic to $\mathscr{M}^{p^{\prime}}(G)$ we will restrict attention to $1<p \leq 2$. It is known that $\hat{\mu} \in \mathscr{M}^{p}(G)$ for every $1 \leq p<\infty$ and every finite regular Borel measure $\mu$ on $G$. As a general reference for $p$-multipliers, see [9].

The spectrum $\sigma(T)$, of an operator $T \in \mathscr{L}\left(L^{p}(G)\right)$, is defined by

$$
\sigma(T):=\left\{\lambda \in \mathbb{C}: T-\lambda I \text { is not invertible in } \mathscr{L}\left(L^{p}(G)\right)\right\} .
$$

For elements $T_{\psi} \in O_{p}(G)$, a basic fact is that

$$
\overline{\psi(\Gamma)} \subseteq \sigma\left(T_{\psi}\right)
$$

for every $1 \leq p<\infty$, [13, Lemma 2.1], where the bar denotes closure in $\mathbb{C}$. Fundamental work of Igari [7] and Zafran [14] established that (1) fails to be an equality in general, even for such a 'nice' group as $G=\mathbb{T}$ (the circle group) and for every $1<p<2$. Indeed, there even exist elements $\psi \in \mathscr{M}^{p}(\mathbb{T}) \cap c_{0}(\mathbb{Z})$ which fail to satisfy (1).

We will say that $\psi \in \mathscr{M}^{p}(G)$ satisfies the spectral mapping property if (1) is an equality. The class of decomposable operators (in an arbitrary Banach space) was introduced by Foiaş [5]; see also [2]. In [1], Albrecht made a detailed study of decomposability for the particular class of $p$-multiplier operators acting in lca groups; see also [4]. Using a functional calculus approach and local spectral theory he showed that the class of decomposable $p$-multiplier operators is rather extensive and, most importantly, that all such operators must satisfy the spectral mapping property, [1, Lemma 3.2].

The purpose of this note is to present criteria of a rather different nature, which ensure the spectral mapping property. The criteria are based directly on geometric, topological and/or algebraic properties of the spectrum itself. Given a compact set $K \subseteq \mathbb{C}$, let $\mathscr{I}(K)$ denote the set of all isolated points of $K$. We can now state the main result. 
THEOREM 1.1. Let $G$ be a compact abelian group, $1<p \leq 2$ and $\psi \in \mathscr{M}^{p}(G)$.

(i) $\psi$ satisfies the spectral mapping property if and only if $\bar{\psi}$ does.

(ii) If $\sigma\left(T_{\psi}\right)=\overline{\mathscr{I}\left(\sigma\left(T_{\psi}\right)\right)}$, then $\psi$ satisfies the spectral mapping property.

(iii) Suppose that $\sigma\left(T_{\psi}\right)$ is totally disconnected. Then $T_{\psi}$ is decomposable and hence, satisfies the spectral mapping property. This is the case if either:

(a) $\sigma\left(T_{\psi}\right)$ is countable.

(b) $\sigma\left(T_{\psi}\right)$ is independent, as a subset of the abelian group $\mathbb{R}^{2}$.

(c) $\sigma\left(T_{\psi}\right)$ is a Kronecker set.

(iv) If $\overline{\psi(\Gamma)}$ is totally disconnected, then $T_{\psi}$ satisfies the spectral mapping property if and only if $T_{\psi}$ is decomposable.

(v) Let $\mu$ be a finite regular Borel measure on $G$ such that $\overline{\hat{\mu}(\Gamma)}$ has capacity zero. Then $\hat{\mu}$ satisfies the spectral mapping property.

(vi) The following statements are equivalent:

(a) $T_{\psi}$ fails the spectral mapping property.

(b) $\sigma\left(T_{\psi}\right) \backslash \overline{\psi(\Gamma)}$ is an uncountable set.

(c) $\overline{\sigma\left(T_{\psi}\right) \backslash \overline{\psi(\Gamma)}}$ is a non-empty perfect set.

The proof is via a series of steps.

For $T \in \mathscr{L}\left(L^{p}(G)\right)$, let $\sigma_{p t}(T), \sigma_{r}(T)$ and $\sigma_{c}(T)$ denote the point, residual and continuous spectra of $T$, respectively, in which case the three sets are pairwise disjoint and have union equal to $\sigma(T)$, [3, page 580].

LeMmA 1.2. Let $1<p \leq 2$ and $\psi \in \mathscr{M}^{p}(G)$. Then

$$
\sigma_{c}\left(T_{\bar{\psi}}\right)=\left\{\bar{\lambda}: \lambda \in \sigma_{c}\left(T_{\psi}\right)\right\}
$$

and also

$$
\sigma_{p t}\left(T_{\bar{\psi}}\right)=\left\{\bar{\lambda}: \lambda \in \sigma_{p t}\left(T_{\psi}\right)\right\}=\bar{\psi}(\Gamma) .
$$

In particular,

$$
\sigma\left(T_{\bar{\psi}}\right)=\left\{\bar{\lambda}: \lambda \in \sigma\left(T_{\psi}\right)\right\}
$$

PrOOF. For any $\varphi \in \mathscr{M}^{p}(G)$, it is routine to check that $T_{\varphi}$ is injective if and only if $0 \notin \varphi(\Gamma)$. It follows easily that

$$
\sigma_{p t}\left(T_{\varphi}\right)=\varphi(\Gamma), \quad \varphi \in \mathscr{M}^{p}(G) .
$$

Putting $\varphi$ equal to $\psi$ and $\bar{\psi}$ in (5), we can deduce (3).

Suppose that $\lambda \in \sigma_{c}\left(T_{\psi}\right)$. Then (5) implies that $\lambda \notin \psi(\Gamma)$ and hence, $\bar{\lambda} \notin \bar{\psi}(\Gamma)$. For each $\gamma \in \Gamma$, the function $f_{\gamma}: g \mapsto\langle g, \gamma\rangle \cdot(\overline{\psi(\gamma)}-\bar{\lambda})^{-1}$, for $g \in G$, belongs to $L^{p}(G)$ and satisfies $\left(T_{\bar{\psi}}-\bar{\lambda} I\right) f_{\gamma}=h_{\gamma}$, where $h_{\gamma}(g):=\langle g, \gamma\rangle$, for $g \in G$. This 
shows that all trigonometric polynomials belong to the range of $T_{\bar{\psi}}-\bar{\lambda} I$ and hence, that this range is dense in $L^{p}(G)$. Accordingly, $\bar{\lambda} \in \sigma_{c}\left(T_{\bar{\psi}}\right)$ which shows that the right-hand side of (2) is contained in $\sigma_{c}\left(T_{\bar{\psi}}\right)$. The reverse inclusion is established similarly.

Since $\sigma_{r}\left(T_{\psi}\right)=\emptyset=\sigma_{r}\left(T_{\bar{\psi}}\right),[13$, Lemma 2.4], (4) follows.

Given any $\psi \in \mathscr{M}^{p}(G)$ it is routine to verify that

$$
\{\bar{\lambda}: \lambda \in \overline{\psi(\Gamma)}\}=\{\mu: \mu \in \overline{\bar{\psi}(\Gamma)}\} .
$$

This observation, together with Lemma 1.2, imply (i) of Theorem 1.1.

To establish Theorem 1.1 (ii) we require the fact that every isolated point of $\sigma\left(T_{\psi}\right)$ belongs to $\sigma_{p t}\left(T_{\psi}\right)$. But, since all elements of $\mathscr{M}^{p}(G)$ are continuous on the discrete space $\Gamma$, this follows from [13, Theorem 2.3]. In view of (5), we can conclude that

$$
\mathscr{I}\left(\sigma\left(T_{\psi}\right)\right) \subseteq \psi(\Gamma) \subseteq \sigma\left(T_{\psi}\right), \quad \psi \in \mathscr{M}^{p}(G)
$$

Under the particular hypothesis that $\overline{\mathscr{I}\left(\sigma\left(T_{\psi}\right)\right)}=\sigma\left(T_{\psi}\right)$, we see from (6) that $\psi$ satisfies the spectral mapping property. This completes the proof of part (ii).

REMARK. It is straightforward to exhibit compact sets $K \subseteq \mathbb{C}$ which satisfy $\overline{\mathscr{I}(K)}=K$, but $K$ is not totally disconnected. So, (ii) of Theorem 1.1 does not follow from part (iii). Of course, there also exist totally disconnected, compact sets $K$ for which $\overline{\mathscr{I}(K)} \neq K$ (for example, the Cantor set, where $\mathscr{I}(K)=\emptyset$ ).

Concerning the proof of Theorem 1.1 (iii), it is known that decomposability of $T_{\psi}$ always follows from the total disconnectedness of $\sigma\left(T_{\psi}\right)$; see the proof of [10, Lemma 2.2 ] which also applies to arbitrary compact abelian groups $G$.

For the definition of a subset of $\mathbb{R}^{2} \simeq \mathbb{C}$ being an independent set (an algebraic notion) or a Kronecker set, we refer to [11, Chapter 5]. Compact, independent subsets of $\mathbb{R}^{2}$ are always totally disconnected, [11, Theorem 5.2.9], and every Kronecker set is independent, [11, Theorem 5.1.4]. So, (b) and (c) of part (iii) are valid. To verify (a), let $C$ be a connected subset of $\sigma\left(T_{\psi}\right)$. Since metric spaces are completely regular and $C$ is countable, it follows that $C$ is actually a singleton set, [8, page 129]. So, $\sigma\left(T_{\psi}\right)$ is totally disconnected whenever it is countable. This completes the proof of part (iii).

For part (iv), we have seen that decomposability of $T_{\psi}$ always implies the spectral mapping property, even if $\overline{\psi(\Gamma)}$ is not totally disconnected. On the other hand, the spectral mapping property means that $\sigma\left(T_{\psi}\right)=\overline{\psi(\Gamma)}$ and so $\sigma\left(T_{\psi}\right)$ is totally disconnected whenever $\overline{\psi(\Gamma)}$ is totally disconnected. Then part (iii) yields the decomposability of $T_{\psi}$. 
For the notion of capacity, which is relevant to (v), we refer to [12] and the references therein. When $p=2$, it is known that $\sigma\left(T_{\hat{\mu}}\right)=\overline{\hat{\mu}(\Gamma)}$, [14, page 357]. This observation, together with [12, Corollary 2.2], establishes (v). For related criteria which also imply the spectral mapping property see [6, Section 4$]$.

REMARK. If $\Gamma$ is discrete and countable (that is, $G$ is metrizable) and $\mu$ is a finite regular Borel measure on $G$ with $\hat{\mu} \in c_{0}(\Gamma)$, then $\overline{\hat{\mu}(\Gamma)}$ is countable and hence, has capacity zero. It follows from (v) of Theorem 1.1 that $\hat{\mu}$ satisfies the spectral mapping property for all $1<p<\infty$; see also [12, page 309]. The condition $\hat{\mu} \in c_{0}(\Gamma)$ is not necessary for this conclusion to hold, [12, Examples 2 and 3]. There also exist $\mu$ (even on $\mathbb{T}$ ) which satisfy the spectral mapping property for all $1<p<\infty$ but, $\overline{\hat{\mu}(\Gamma)}$ is uncountable, [12, pages 310-312].

Finally, part (vi) of Theorem 1.1 follows from (6) and the following result (with the choice $J:=\overline{\psi(\Gamma)}$ and $\left.K:=\sigma\left(T_{\psi}\right)\right)$.

LEMMA 1.3. Let $K \subseteq \mathbb{C}$ be non-empty and compact.

(i) $\mathscr{I}(K)$ is a countable set, possibly empty.

(ii) $K$ is countable if and only if the set $\mathscr{A}(K)$ of all accumulation points of $K$ is countable.

(iii) Let $J$ be a closed subset of $K$ with $\mathscr{I}(K) \subseteq J$. Then either $J=K$ or $\overline{K \backslash J}$ is a non-empty perfect set (that is, $\overline{K \backslash J}=\mathscr{A}(\overline{K \backslash J})$ ). If $J \neq K$, then $K \backslash J$ (hence, also $\overline{K \backslash J}$ ) is uncountable.

PROOF. (i) $K$ is a separable metric space and so has a countable base for its topology. Since each set $\{x\}$, for $x \in \mathscr{I}(K)$, belongs to this base, it follows that $\mathscr{I}(K)$ is countable.

(ii) Since $K$ is the disjoint union of $\mathscr{I}(K)$ and $\mathscr{A}(K)$, (ii) follows from (i).

(iii) Suppose that $\overline{K \backslash J} \neq \emptyset$ and let $x \in \overline{K \backslash J}$.

If $x \notin K \backslash J$, then $x \in \mathscr{A}(K \backslash J) \subseteq \mathscr{A}(\overline{K \backslash J})$.

If $x \in K \backslash J$, then $K \backslash J$ being open in $K$ ensures the existence of a ball $B_{x}$ (centre $x$ and positive radius) which is open in $K$ and satisfies $B_{x} \subseteq K \backslash J$. Since $\mathscr{I}(K) \subseteq J$ it follows that $x \in \mathscr{A}(K)$. Choose any sequence $\left\{x_{n}\right\}_{n=1}^{\infty}$ in $K \backslash\{x\}$ which converges to $x$. Then all but finitely many of the $x_{n}$ must belong to $B_{x}$. Remove these finitely many points leaves a sequence in $(K \backslash J) \backslash\{x\}$ which converges to $x$. Accordingly, $x \in \mathscr{A}(K \backslash J) \subseteq \mathscr{A}(\overline{K \backslash J})$.

This establishes that $\overline{K \backslash J}$ is a perfect set whenever $J \neq K$.

Suppose now that $J \neq K$. The set $K \backslash J$ is open in $K$ and each singleton set $\{x\}$, for $x \in K \backslash J$, is nowhere dense in $K$ (because $\mathscr{I}(K) \subseteq J$ ). So, if $K \backslash J$ is countable, then it is of first category in $K$. By Baire's Theorem $J=K \backslash(K \backslash J)$ would be dense 
in $K$, that is, $K=\bar{J}(=J)$ contrary to the assumption that $J \neq K$. Hence, $K \backslash J$ is uncountable.

As a concluding remark, we point out that Theorem 1.1 (vi) is an extension of a result of Zafran, [13, Lemma 2.6], proved for $\psi \in \mathscr{M}^{p}(G) \cap c_{0}(\Gamma)$. Our result shows that the condition $\psi \in c_{0}(\Gamma)$ can be omitted.

\section{References}

[1] E. Albrecht, 'Decomposable systems of operators in harmonic analysis', in: Toeplitz Memorial Conference in Operator Theory (ed. I. Gohberg), Oper. Theory Adv. Appl. 4 (Birkhäuser, Basel, 1982) pp. 19-35.

[2] I. Colojoară and C. Foiaş, Theory of generalized spectral operators (Gordon and Breach, New York, 1968).

[3] N. Dunford and J. T. Schwartz, Linear operators l: General theory (Interscience, New York, 1964).

[4] J. Eschmeier, 'Operator decomposability and weakly continuous representations of locally compact abelian groups', J. Operator Theory 7 (1982), 201-208.

[5] C. Foiaş, 'Spectral maximal spaces and decomposable operators', Arch. Math. (Basel) 14 (1963), 341-349.

[6] C. C. Graham, K. E. Hare and D. L. Ritter, 'The size of $L^{p}$-improving measures', J. Funct. Anal. 84 (1989), 472-495.

[7] S. Igari, 'Functions of $L^{p}$ multipliers', Tôhoku Math. J. 21 (1969), 304-320.

[8] K. Kuratowski, Topology II (PWN-Polish Scientific Publ., Warszawa, 1968).

[9] R. Larsen, An introduction to the theory of multipliers (Springer, Berlin, 1971).

[10] G. Mockenhaupt and W.J. Ricker, 'Fuglede's theorem, the bicommutant theorem and $p$-multiplier operators for the circle', J. Operator Theory 49 (2003), 295-310.

[11] W. Rudin, Fourier analysis on groups (McGraw-Hill, New York, 1973).

[12] P. Sarnak, 'Spectra of singular measures as multipliers on $L^{p}$ ', J. Funct. Anal. 37 (1980), 302-317.

[13] M. Zafran, 'On the spectra of multipliers', Pacific J. Math. 47 (1973), 609-626.

[14] _ 'The spectra of multiplier transformations on the $L_{p}$-spaces', Ann. of Math. (2) 103 (1976), 355-374.

Math.-Geogr. Fakultät

Katholische Universität Eichstätt-Ingolstadt

D-85072 Eichstätt

Germany

e-mail: werner.ricker@ku-eichstaett.de 